\title{
Towards Closing the Gap in Weakly Supervised Semantic Segmentation with DCNNs: Combining Local and Global Models
}

\author{
Christoph Mayer \\ ETH Zürich, Switzerland \\ chmayerevision.ee.ethz.ch
}

\author{
Radu Timofte \\ ETH Zürich, Switzerland \\ timofer@vision.ee.ethz.ch
}

\author{
Grégory Paul \\ ETH Zürich, Switzerland \\ grpaul@vision.ee.ethz.ch
}

\begin{abstract}
Generating training sets for DCNNs is a bottleneck for modern real-world applications. This is a demanding task for applications where annotating training data is costly, such as in semantic segmentation. In the literature, there is still a gap between the performance achieved by a network trained on full and on weak annotations. In this paper, we establish a strategy to measure this gap and to identify the ingredients necessary to reduce it.

On scribbles, we establish new state-of-the-art results: we obtain a mIoU of $75.6 \%$ without, and $75.7 \%$ with CRF post-processing. We reduce the gap by $64.2 \%$ whereas the current state-of-the-art reduces it only by $57.5 \%$. Thanks to a systematic study of the different ingredients involved in the weakly supervised scenario and an original experimental strategy, we unravel a counter-intuitive mechanism that is simple and amenable to generalisations to other weaklysupervised scenarios: averaging poor local predicted annotations with the baseline ones and reuse them for training a DCNN yields new state-of-the-art results.
\end{abstract}

\section{Introduction}

Semantic segmentation aims at extracting semantically meaningful segments and classify each part into one of the classes predefined by the user. It is a central problem to computer vision, because it bridges a lower-level task (image segmentation) to a higher-level one (scene understanding). State-of-the-art models are data-driven and require for training examples of images together with the segmentation of the intended classes. Recently, Deep Convolutional Neural Networks (DCNNs) have achieved the best performance to date on the public data sets used for comparing different frameworks in a normalized fashion [8], such as PASCAL VOC [6] or MS-COCO [19].

However, DCNNs are greedy in the amount of training data. For semantic segmentation, providing a training set is a demanding task, because it requires assigning carefully a label to each pixel in the training set. This poses two problems for real-world applications of semantic segmentation: versatility and scalability. Versatility is an issue when the classes of interest differ from the ones in the training set: this requires re-annotating the training images. Scalability is an issue when the number of training images grows significantly, i.e. at the scale of data sets that are nowadays available and required in real-world applications. A solution to these issues is to rely on weak supervision.

\subsection{Weakly-supervised semantic segmentation}

In semantic segmentation, a full annotation holds information about the location, the shape, the spatial relationships between segments, the co-occurrence of classes, the class of each segment, etc. In contrast, weak annotations do not provide direct examples of semantic segments, but offer only partial cues: image-level tags provide class information $[23,11]$; point supervision provides class and approximate location [1], bounding boxes [12] provide class, approximate location and extent; scribbles [18, 35, 32] provide class, approximate object location and extent. In addition to things (i.e. with a distinct size and shape, e.g. cars, people), scribbles can also annotate stuff (i.e. with no specific spatial extent or shape, e.g. road, sky), see [7]. On one hand, Weak Annotations (WAs) are easier to collect (see [1] for timings), more versatile, and better for upscaling the training set. On the other hand, WAs are not exhaustive and subject to human annotation errors.

Therefore, weak supervision requires specific training strategies. Different strategies exist in the literature. Hong et al. [10] adapt the DCNN architecture. Designing new loss functions to promote weak annotations to full ones is popular: [1] use objectness for point supervision and other higher-level priors, and [32] designed a new loss function inspired by "shallow" segmentation. Post-processing weak annotations to full ones as an intermediate step to train an existing DCNN is also popular: bounding boxes propagated by GrabCut [22] or other strategies [12], scribbles extended by super-pixels and a variational model [18]. 


\section{2. (Semi-)interactive segmentation}

(Semi-)interactive segmentation is a boundary case of the weakly-supervised setting where the training set is reduced to a single image. State-of-the-art interactive segmentation frameworks are in essence Bayesian and variational. In the interactive setting, the amount of training data is low (reduced to the user inputs, such as bounding boxes [25], scribbles [34, 28], etc.) and makes the prediction for the unlabelled pixels uncertain. Therefore, Bayesian models are an attractive paradigm for this task, as they allow incorporating prior knowledge (e.g. boundary length [21], spatial semantic relation [5] and co-occurrence [15], appearance and smoothness in color space [13]) that facilitates the predictions when the semantic model alone is uncertain.

\subsection{Goals and Contributions}

In this work we tackle the problem of training a DCNN for the semantic segmentation problem in a weakly supervised setting. See [9] for a recent review and the references therein. Our main focus is on scribble annotations for which annotation data are available for the PASCAL VOC data set [18]. Among the possible strategies described in Sec. 1.1, we follow the strategy consisting in post-processing the weak annotations to generate full annotations to train a subsequent DCNN from scribbles.

Our goal is to identify simple ingredients to reduce the gap between the baseline accuracy achievable by training the network on the weak annotations only (lower bound) and the accuracy obtained by training on the fully annotated training set (upper bound).

Our comprehensive experimental design (Tab. 1) allowed us to identify an unexpected interaction between local and global Predictive Annotator Models (PAMs) that conspire to boost the overall accuracy (Fig. 2) and establish new state-of-the-art results (Tab. 2).

\section{Methods}

\subsection{Overview of the experimental strategy}

Weak supervision strategies. Fig. 1 displays the different levels of training required in a fully and a weakly supervised scenario. In both scenario, the training of the segmentation DCNN requires Full Annotations (FAs). For full supervision, FAs are directly provided by humans, hence called Human Full Annotations (HFAs).

For weak supervision, WAs need to be extended to FAs. This can be achieved in two ways. The simplest way is to introduce an additional label that is used for unlabelled pixels, and define the segmentation cost $\mathcal{L}_{\text {seg }}$ at these labels to be 0 . This amounts to training only on the annotated pixels data. This defines a baseline training strategy because it corresponds to a default strategy that does not attempt to

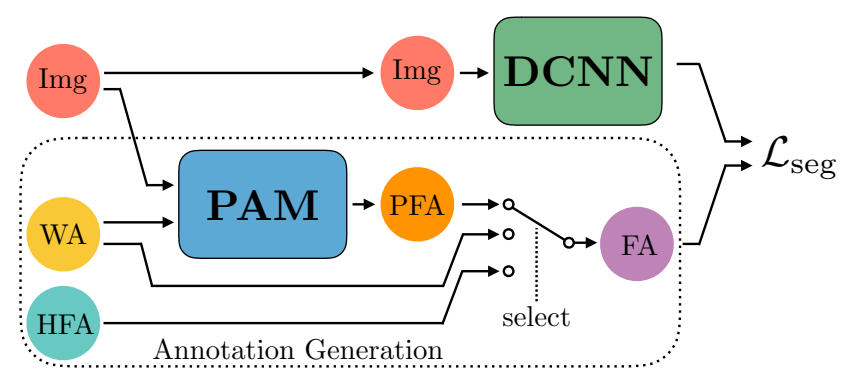

Figure 1. Training a Segmentation DCNN in a fully- and weakly-supervised scenario. The segmentation cost $\mathcal{L}_{\text {seg }}$ requires both training images (Img) and Full Annotations (FA). In a fully supervised scenario, FAs are produced by humans (HFA: Human FA). In a weakly supervised scenario, humans produce only weak annotations (WA). WAs can be used directly for training as FAs by using a special label at the unlabelled pixels, e.g. None and define accordingly $\mathcal{L}_{\text {seg }}$ to be 0 at these pixels. WAs can also be used to train an intermediate Predictive Annotator Model (PAM) that will predict the classes for the missing annotations. PAMs can be trained independently for each training image (local PAM), or trained on the whole training set (global PAM).

predict the unlabelled pixels and that makes the minimum modifications to the segmentation cost.

Another strategy amounts to use the WAs to train an intermediate model to predict the semantic classes at the unlabelled locations, resulting in a Predicted Full Annotation (PFA). This is called a Predictive Annotator Model (PAM).

Defining the Gap between full and weak supervision. Our goal is to unravel the ingredients required to train a DCNN on WAs that achieves performances comparable to the same DCNN trained on HFAs. We define the gap as the segmentation accuracy difference between the network trained in the full and in the weak supervision scenario. If we use Mean Intersection over Union (mIoU) as the accuracy measure, this writes:

$$
\text { Gap }:=\mathrm{mIoU}^{\text {Full }}-\mathrm{mIoU}^{\text {Weak }} .
$$

We assess a given training strategy by computing the relative reduction of the Gap compared to the baseline strategy.

Assessing PAMs. PAMs introduce an intermediate level of training. We evaluate their prediction quality by reporting the predicted annotations accuracy (column PFA in Tab. 1). In addition, we also report the accuracy of the DCNN after training (sub-column Train below DCNN in Tab. 1). This is usually omitted in the literature tackling the full supervision problem because the predictions of the trained network on the training set will automatically be worse than the original annotations.

However it is unclear how the accuracy of the predicted annotations will compare to the predictions of the network 
after training because they correspond to two levels of training. In addition, this comparison is important to study how improvements in the predicted annotation accuracy (measured on the training set) translate into improvements of the segmentation accuracy of the resulting trained network (measured on the validation set).

\subsection{Data and weak annotations}

Following [18, 32, 33], we use the PASCAL VOC data set [6] with the publicly available scribble WAs [18].

Curated WAs: assessing human annotation errors. Inevitably, weak annotations contain errors. For scribbles, two sources are possible: assigning the wrong class or annotating multiple classes with one stroke. To assess the impact of human annotation errors in the weakly supervised scenario, we assemble a curated training set. We keep the scribble positions but we assign the ground truth semantic class to each annotated pixel. Furthermore, we require that, for each image, the human annotator has labelled all the classes present in the ground truth. We drop the images not satisfying this requirement: the curated training set contains 10489 instead of 10582 images.

We always report the results about the best strategy for both the curated and the original scribbles (Tab. 1, lines 8/9 and 12/13, Tab. 2). However, for the sake of simplicity, we show the results about the different ingredients in the training strategy that lead us to identifying our best strategy only on the curated dataset (Tab. 1, lines 3-9 and Fig. 2).

\subsection{Predictive annotator models}

PAMs can be trained for each training image independently (many local PAMs) or for the whole training set (one single global PAM).

\subsubsection{Local PAM: random forest}

In interactive image segmentation two PAMs are common: Gaussian Mixture Models (GMMs) [25, 20] and Random Forest (RF) classifiers [3, 27]. We use RF instead of GMM because RFs train quickly even with high dimensional data and provides a feature importance score enabling feature ranking. We use the custom Gini feature importance score available in python in scikit-learn. The features found in DCNNs trained for classification display desirable properties such as compositionality, invariance and class discrimination for ascending layers [38]. The first-layer contain low-level image filters for different colour patterns e.g. edge or corner filters. Thus, we propose using the firstlayer features of VGG-16 [29] and AlexNet [14] to train the RF classifier on each training image. We use 50 trees and we apply the feature importance score to select the 100 most informative ones out of 160. Santner et al. [27, 28] use 30, 100 and 250 trees. We have observed that using 50 trees and 100 features leads to finer-grained predictions and decreases the amount of strong false positives and false negatives, i.e. class predictions with probability estimates of 0.0 or 1.0, preventing further improvement by regularisation.

\subsubsection{Global PAM: DeepLab}

A state-of-the-art DCNN in supervised semantic segmentation is DeepLab [4]. Different versions of DeepLab are used in weakly-, semi-, or fully-supervised settings [18, 22]. We propose to use a simplified version of DeepLabV2 as the global PAM. We avoid pre-training DeepLabV2 on any segmentation dataset such as MS-COCO [4]. For a detailed description of the exact architecture, training strategy and hyper parameter optimization, we refer the reader to the supplementary material.

\subsection{Regularising PAMs}

A Bayesian semantic segmentation model consists of a semantic model encoding how well pixels fit in their putative classes (encoded in a data-fitting term) and a consistency model encoding how well a particular segmentation fits some desired prior knowledge (encoded in a regularisation term). User inputs are used to train the semantic model derived from the PAMs (described in Sec. 2.3) that learn how pixels should be labelled according to the human annotator. Regularisation helps when the semantic model is uncertain about how to assign a class to a pixel, in particular when the amount of pixels to calibrate the semantic model is low. In this work, we compare two popular variational models: Potts [21] and Fully Connected Conditional Random Field (FC-CRF) [13]. Both share the same data-fitting term, but differ in their regularisation.

Bayesian data-fitting terms. The data-fitting term writes as the sum over the pixels (denoted $\mathcal{I}$ ) of the scalar product between the semantic segmentation mask vector, denoted $M_{i}$, and the negative log -labelling probability vector, denoted $\boldsymbol{P}_{i}$, see for example [21]:

$$
\mathcal{E}_{\mathrm{d}}(\boldsymbol{P}, \boldsymbol{M}):=\sum_{i \in \mathcal{I}}\left\langle-\log \boldsymbol{P}_{i}, \boldsymbol{M}_{i}\right\rangle .
$$

The vector $\boldsymbol{P}_{i}$ encodes the semantic segmentation model: $\boldsymbol{P}_{i c}$ is the probability of assigning class $c \in \mathcal{C}$ at pixel $i$. The vector $M_{i}$ represents a valid semantic segmentation hypothesis, i.e. an element of the unit probability simplex. A mask vector containing only zeroes and ones corresponds to a proper labelling, i.e. a unique label is assigned everywhere. Otherwise, the segmentation is called soft, and a proper labelling is recovered by selecting at each pixel $i$ the class with highest value in $M_{i}$. 
The probability vector $\boldsymbol{P}$ is derived from the PAMs (see Sec. 2.3) by normalising appropriately their soft predictions in the probability simplex. They are denoted $\boldsymbol{P}^{\text {local }}$ and $\boldsymbol{P}^{\text {global }}$ for the local and global PAMs respectively.

Potts regularisation. The Potts model penalises the total length of the interface between classes. It is popular for its simplicity and the various efficient algorithms to compute the associated maximum a posteriori (MAP) (see [21]). This regularisation amounts to summing the weighted total variation of the mask for each class:

$$
\mathcal{E}_{\mathrm{r}}^{\mathrm{TV}}(\boldsymbol{M}):=\lambda \sum_{c \in \mathcal{C}} \operatorname{TV}_{g}\left(\boldsymbol{M}_{c}\right),
$$

where $g$ is the edge-stop function driving the class boundaries towards high image gradients. It is defined as $g_{i}=$ $\exp \left(-\eta|\nabla \boldsymbol{I}|_{i}\right)$.

FC-CRF regularisation. For comparison, we also use the more complex regularisation of the FC-CRF model [13]. It is popular in the literature as a post-processing to recover detailed features from the blobby predictions of a DCNN. This regulariser is defined as

$$
\mathcal{E}_{\mathrm{r}}^{\mathrm{CRF}}(\boldsymbol{M}):=\sum_{c \in \mathcal{C},(i, j) \in \mathcal{I}^{2}}\left[\boldsymbol{M}_{i} \neq \boldsymbol{M}_{j}\right]\left(w_{1} \boldsymbol{A}_{i j}+w_{2} \boldsymbol{S}_{i j}\right),
$$

where $\boldsymbol{A}_{i j}:=\exp \left(-d_{i j}^{2} / 2 \sigma_{\alpha}^{2}-\delta_{i j}^{2} / 2 \sigma_{\beta}^{2}\right)$ and $\boldsymbol{S}_{i j}:=$ $\exp \left(-d_{i j}^{2} / 2 \sigma_{\gamma}^{2}\right)$ are the appearance and smoothness kernel respectively, with $d_{i j}$ the Euclidean distance between pixels $i$ and $j$, and $\delta_{i j}$ the Euclidean distance in color space between pixels $i$ and $j$. This prior tends to group nearby pixels with similar colours (appearance kernel), and at the same time penalise small clusters (smoothness kernel).

MAP annotations prediction. For both models we solve the associated MAP problem

$$
\mathrm{M}^{\mathrm{MAP}}(\boldsymbol{P}):=\underset{\boldsymbol{M} \in \Delta}{\arg \inf } \mathcal{E}_{\mathrm{d}}(\boldsymbol{P}, \boldsymbol{M})+\mathcal{E}_{\mathrm{r}}(\boldsymbol{M}),
$$

with $\Delta:=\bigcup \Delta_{i}$ the set of valid soft segmentation masks, and $\Delta_{i}$ the probability simplex defined at pixel $i$. The MAP problem aims at finding the optimal trade-off between fitting the data, driven by $\mathcal{E}_{\mathrm{d}}$ (see Eq. (2)) and the regularisation $\mathcal{E}_{\mathrm{r}}$, being either Potts (see Eq. (3)) or FC-CRF (see Eq. (4)).

We solve the Potts MAP problem using the strategy described in [24]. For the FC-CRF MAP problem, we use the implementation of [13] found at [2]. We refer the reader to the supplementary material for a detailed account of the procedure to select the regularisation parameters and the model-dependent parameters.
We denote by $\boldsymbol{M}^{\text {local }}:=\mathrm{M}^{\mathrm{MAP}}\left(\boldsymbol{P}^{\text {local }}\right)$ and $\boldsymbol{M}^{\text {global }}:=$ $\mathrm{M}^{\mathrm{MAP}}\left(\boldsymbol{P}^{\text {local }}\right)$ the regularisation of the local and the global PAMs respectively.

\subsection{Predicted annotations from PAMs}

For each training image, the Predicted Full Annotations are obtained at each pixel $i$ independently from a soft segmentation $\boldsymbol{S} \in \Delta$ by:

$$
\operatorname{PFA}_{i}(\boldsymbol{S}):=\underset{c \in \mathcal{C}}{\arg \max } \boldsymbol{S}_{i} .
$$

Without regularisation, we define the local and the global PFAs by

$$
\begin{aligned}
\mathrm{PFA}^{\text {local }} & :=\operatorname{PFA}\left(\boldsymbol{P}^{\text {local }}\right) \\
\mathrm{PFA}^{\text {global }} & :=\operatorname{PFA}\left(\boldsymbol{P}^{\text {global }}\right) .
\end{aligned}
$$

We define the regularised PFAs by

$$
\begin{aligned}
\mathrm{PFA}^{\text {local+MAP }} & :=\operatorname{PFA}\left(\boldsymbol{M}^{\text {local }}\right) \\
\mathrm{PFA}^{\text {global+MAP }} & :=\operatorname{PFA}\left(\boldsymbol{M}^{\text {global }}\right) .
\end{aligned}
$$

\section{Experiments and results}

To explore and identify weak supervision strategies we use DeepLabV2 both as the global annotator model and as the segmentation DCNN (see Fig. 1 and Sec. 2.1). However, after having identified the best strategy, we test different networks and architectures for the segmentation DCNN in Sec. 3.5. We report the corresponding values in Tab. 1, 2 and Fig. 2.

\subsection{Measuring the Gap}

Under full supervision, DeepLabV2 achieves a mIoU of 71.5 on the validation set, which is consistent with the performances reported for the TensorFlow implementation $[36,37]$.

Under weak supervision (see Sec. 2.1), DeepLabV2 trained solely on the original scribbles found in [18] achieves a mIoU of 64.3 on the validation set, establishing a gap of 7.2. As expected, on the curated annotations, the mIoU is higher (67.1), and the gap smaller (4.4), see Tab. 1. This gives a first insight on the adversarial impact of the errors in the WA on the resulting trained model.

We explore different weak supervision strategies on the curated annotations because the gap is smaller due to a baseline model that has already a high mIoU (67.1). This makes it harder for any strategy to be elected. However, for our best weak supervision strategy, we always report the results on the original scribbles dataset, because in practice curated annotations do not exist, see Tab. 1. In addition, when comparing our results to others in Tab. 2, we always use the original scribble dataset of [18]. 
Table 1. Towards closing the gap. All the values correspond to mIoU [\%] except the last column. The Full Gap corresponds to the gap between the two methods shown in light grey, for both the curated and the original WAs [18]. The Remaining Gap defines the difference between DeepLabV2 trained on human or predicted full annotations. Gap Reduction (\%) is defined by (Full Gap-Remaining Gap)/Full Gap. The colored symbols correspond to Fig. 2. The column PFA reports the mIoU of the PFAs of the training images produced by the PAMs.

\begin{tabular}{|c|c|c|c|c|c|c|c|c|c|c|c|c|}
\hline \multicolumn{2}{|c|}{ Training Data } & \multicolumn{2}{|c|}{ PAM } & \multicolumn{2}{|c|}{ Regularisation } & \multirow{2}{*}{$\begin{array}{r}\text { Marker } \\
\text { Fig. } 2\end{array}$} & \multirow{2}{*}{$\begin{array}{l}\text { PFA } \\
\text { Train }\end{array}$} & \multicolumn{2}{|c|}{ DCNN } & \multicolumn{3}{|c|}{ Gap } \\
\hline & & Local & Global & Potts & FC-CRF & & & Train & Val & Full & Remaining & Reduction (\%) \\
\hline \multirow{9}{*}{ 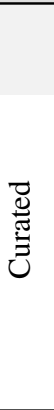 } & HFA & & & & & & 100 & 80.7 & 71.5 & n.a. & n.a. & n.a. \\
\hline & WA & & & & & 0 & 100 & 74.8 & 67.1 & n.a. & n.a. & n.a. \\
\hline & & $\checkmark$ & & & & 0 & 61.5 & 67.8 & 61.0 & 4.4 & 10.5 & $-138.6 \%$ \\
\hline & & $\checkmark$ & & $\checkmark$ & & $\mathbf{\square}$ & 73.3 & 70.9 & 63.3 & 4.4 & 8.2 & $-70.4 \%$ \\
\hline & & & $\checkmark$ & $\checkmark$ & & $\mathbf{\square}$ & 79.4 & 74.6 & 67.8 & 4.4 & 3.7 & $15.9 \%$ \\
\hline & PFA & & $\checkmark$ & & $\checkmark$ & $\Delta$ & 79.6 & 75.6 & 68.2 & 4.4 & 3.3 & $25.0 \%$ \\
\hline & & $\checkmark$ & $\checkmark$ & & & - & 81.0 & 76.4 & 69.2 & 4.4 & 2.3 & $47.7 \%$ \\
\hline & & $\checkmark$ & $\checkmark$ & $\checkmark$ & & $\mathbf{\square}$ & 84.2 & 76.8 & 69.7 & 4.4 & 1.8 & $59.1 \%$ \\
\hline & & $\checkmark$ & $\checkmark$ & & $\checkmark$ & $\Delta$ & 83.9 & 77.6 & 70.0 & 4.4 & 1.5 & $65.9 \%$ \\
\hline \multirow{4}{*}{ 点 } & HFA & & & & & & 100 & 80.7 & 71.5 & n.a. & n.a. & n.a. \\
\hline & WA & & & & & & 95.4 & 71.7 & 64.3 & n.a. & n.a. & n.a. \\
\hline & PEA & $\checkmark$ & $\checkmark$ & $\checkmark$ & & & 81.6 & 76.4 & 68.8 & 7.2 & 2.7 & $62.5 \%$ \\
\hline & РГА & $\checkmark$ & $\checkmark$ & & $\checkmark$ & & 81.4 & 76.5 & 69.1 & 7.2 & 2.4 & $66.7 \%$ \\
\hline
\end{tabular}

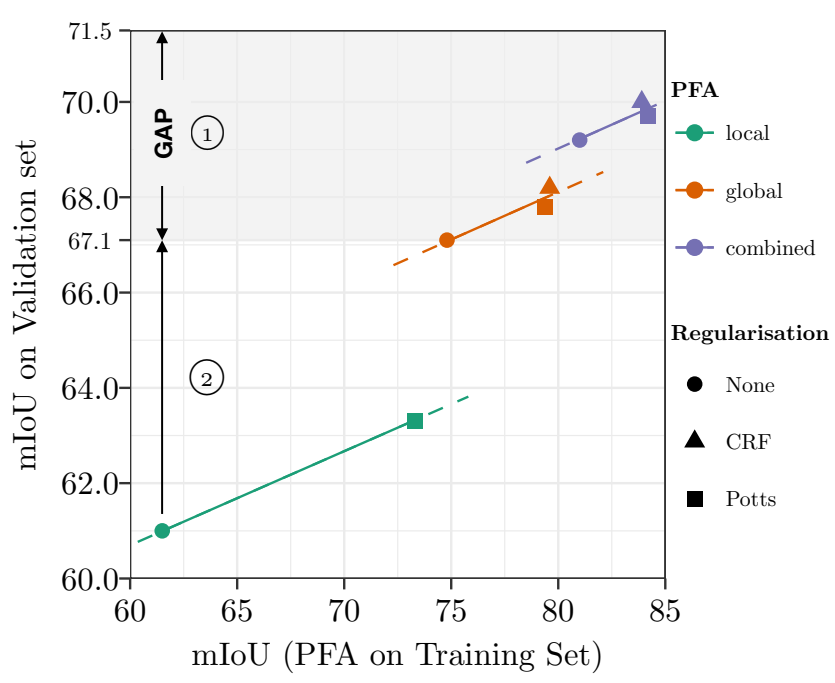

Figure 2. Visual summary of our study. The gap is the difference between DeepLabV2 trained solely on scribbles and DeepLabV2 trained on the human full annotations. See Tab. 1.

\subsection{Local PFAs are worse than the baseline}

We recall that the global annotator model, i.e. the baseline, is DeepLabV2 trained solely on the scribbles (see Sec. 2.1). This represents only $1.6 \%$ of pixels annotated on average per class. We expect that annotating all pixels by training a local annotator model per training image helps. However these additional annotations are predicted, and hence prone to errors.

Assessing the local PFAs. On each training image we train a RF (see Sec. 2.3.1) on the scribbled pixels, and we use it to predict (using Eq. 7) the class for the unlabelled ones. These predicted annotations achieve a mIoU of 61.5 , significantly lower than the baseline that achieves annotations with a mIoU of 74.8. Potts regularisation for each local annotator (Eq. 9) improves the mIoU of the predicted annotations by 11.8 , up to 73.3. It lags behind the baseline only by 1.1. We interpret this result by the fact that the global PAM is trained on all the scribbles, whereas each local PAM is trained only on the scribbles available for the image where it predicts the missing annotations. To some extent, Potts regularisation compensates for this scarce amount of training pixels.

The relationship between PFAs quality and the accuracy after training is nonlinear. A priori, we expect that the better the predicted annotations, the better the segmentation after training. We also expect that for a given improvement in the predicted annotations quality, we obtain a lesser improvement after training of the resulting network.

After training DeepLabV2 on the local PFAs, we obtain on the validation set a mIoU of 61.0 (without regularisation, $\bigcirc$ ) and 63.3 (with regularisation, $\square$ ). As expected, the improvement by 11.3 of the mIoU due to regularisation of the local PFAs, yields after training a lesser improvement of 2.3 on the validation set. Surprisingly, the regularised local PFAs, having an accuracy comparable to the baseline, yield after training an accuracy 3.8 points below the baseline, see Fig. 2.

These findings suggest that the annotation errors predicted by the local and the global PAMs are qualitatively different. Fig. 4 shows examples of images where the local 


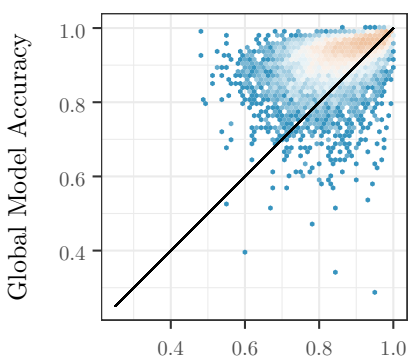

Local Model Accuracy

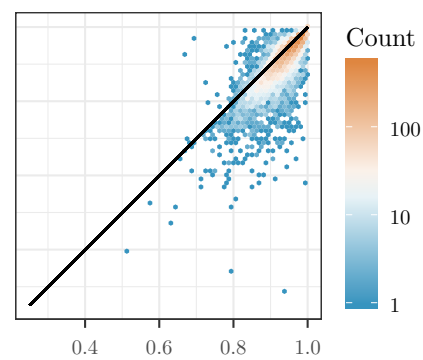

Combined Model Accuracy

Figure 3. Comparing local, global, and combined PFAs. The accuracy is the mIoU. The diagonal is the equal accuracy cutoff.

PAM is worse than the baseline. In such cases, we observe that the local annotators produce scarce and inconsistent labelling, whereas the global annotator yields overall good annotations, but with imprecise boundaries.

However, local models have the potential to capture better, in a given training image, the labelling intended by the human annotator. To quantify this effect we compare the pixel accuracy of the PFAs generated by the local and the global PAMs. In Fig. 3 (left panel), we observe that for most images, the pixel accuracy is best for the baseline (i.e. the global PAM), but a fair amount of images are better labelled by the local PAMs.

\subsection{Regularising global PFAs improves only marginally}

In the previous section, we have learned that local PAMs are not good enough to beat the baseline that is trained solely on scribbles. But we have shown that regularising the predicted annotations can still translate in a significant improvement in the resulting trained network.

Regularising the annotations predicted by the global PAM with Potts ( $\square$ ) boosts the PFAs by 4.6 up to 79.4 in $\mathrm{mIoU}$ and leads to 67.8 after training. Using the more complex FC-CRF regulariser $(\Delta)$ leads to comparable improvements in both the PFAs quality (79.6) and the accuracy of the trained network (68.2).

Regularising the annotation predictions by the baseline does help closing the gap. However, the improvement is only marginal and reduces the gap only by $15.9 \%$ (Potts) and $25.0 \%$ (FC-CRF).

\subsection{Combining local and global PFAs is best}

In Sec. 3.2 we have observed that there are images where the local PAMs achieve better annotations (i.e. below the diagonal in Fig. 3, left panel). This suggests that the local and the global PAMs can be complementary.

To test this idea, we combine them by averaging their
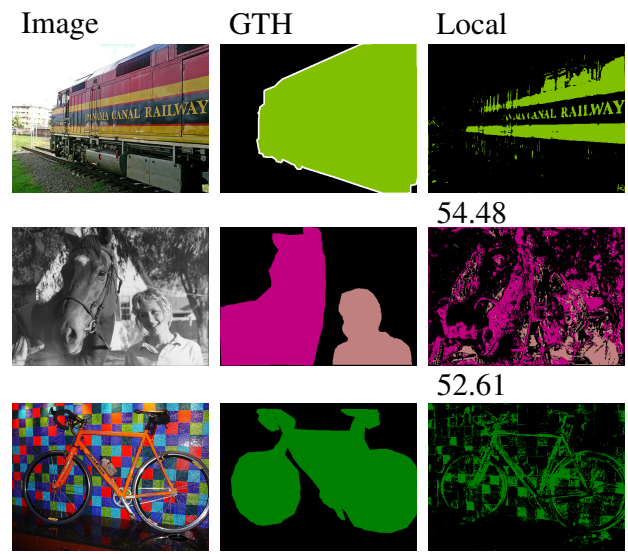

Global
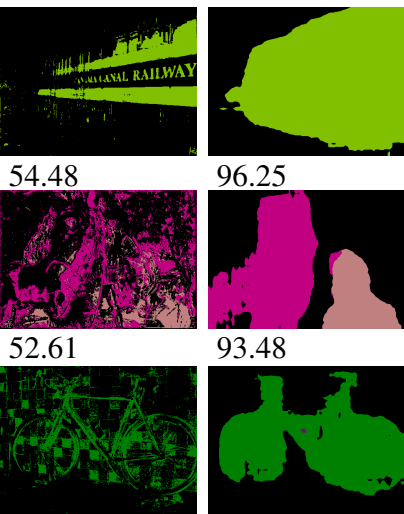

93.48
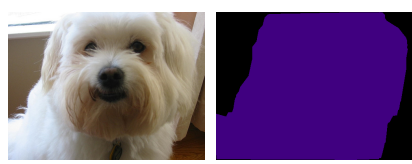

61.58

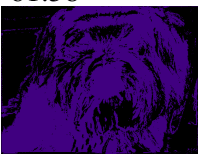

74.03
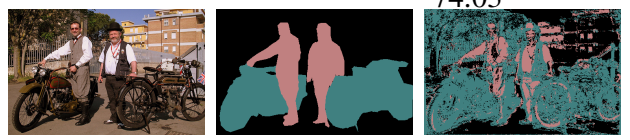

59.83
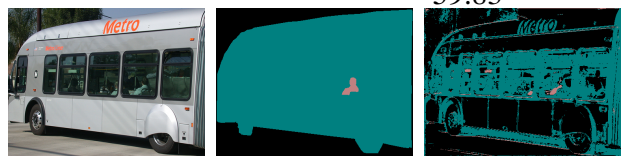

52.73

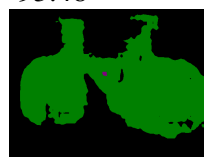

89.51
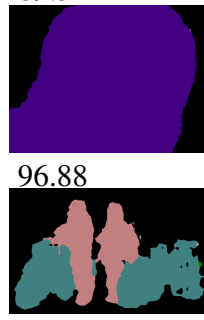

90.64

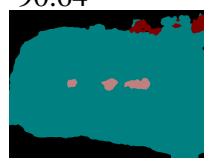

89.56

Figure 4. Examples where global PFAs have a higher pixel accuracy than the local ones.

predictions:

$$
\begin{aligned}
\boldsymbol{P}^{\text {comb }} & :=\frac{1}{2} \boldsymbol{P}^{\text {local }}+\frac{1}{2} \boldsymbol{P}^{\text {global }} \\
\mathrm{PFA}^{\text {comb }} & :=\operatorname{PFA}\left(\boldsymbol{P}^{\text {comb }}\right) .
\end{aligned}
$$

The right panel of Fig. 3 shows that the combined local and global annotation predictions improve overall compared to the global PAM alone.

Fig. 5 shows images where the combined predictions have an accuracy superior to either the local or the global PAM. Furthermore, Fig. 5 shows examples where the global PAM assigns class labels absent in the ground truth. However, a local PAM avoid these errors because they are constrained to predict, in a given training image, only the classes present in the scribble annotations. Therefore, combining local and global PAMs reduces the effect of predicting foreign class labels. These observations support our claim in Sec. 3.2 that the predicted annotations from the local and the global PAMs are qualitatively different.

Without regularisation, PFA $^{\text {comb }}$ achieves a mIoU of 81 (local: 61 and global: 74.8). Subsequently training DeepLabV2 achieves 69.2(๑) on the validation set. Hence, combining local and global PAMs boosts the quality of the 


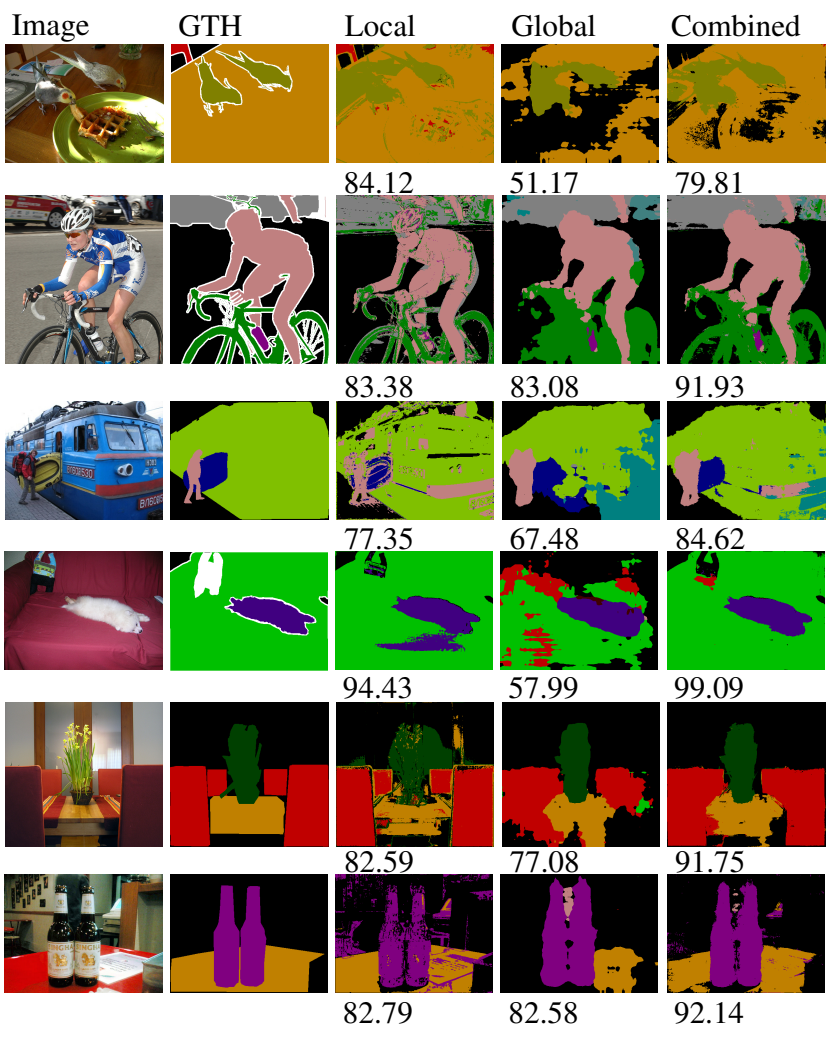

Figure 5. Examples where the combined PFAs have a higher accuracy than either the local or the global ones.

PFAs substantially. The model trained on these improved PFAs reduces the gap by $47.7 \%$ down to 2.3 .

We use the averaged predictions $\boldsymbol{P}^{\text {comb }}$ to obtain the regularised combined predicted annotations :

$$
\mathrm{PFA}^{\mathrm{comb}+\mathrm{MAP}}:=\operatorname{PFA}\left(\mathrm{M}^{\mathrm{MAP}}\left(\boldsymbol{P}^{\mathrm{comb}}\right)\right)
$$

As expected, regularisation improves both the PFAs quality and the accuracy after training, leading to models with a mIoU of $69.7(\square)$ and $70.0(\Delta)$ on the validation set. Hence, combining local and global PAMs by averaging their predictions has a larger impact than regularisation alone. However, in synergy, these two ingredients lead to the best models that reduce the gap by 59\% to 1.8 (Potts) and $65.9 \%$ to 1.5 (FC-CRF).

We make two additional controls for our best strategy: we test our weak supervision strategy on the non-curated original scribbles of [18], and on the test set of the PASCAL VOC2012 challenge. On the non-curated annotations, we achieve even better results and reduce the gap by $62.5 \%$ (Potts) and 66.7\% (FC-CRF). This shows that our strategy is robust to human annotation errors. Additionally, we report the mIoU on the test set, where we achieve 70.4 (FC-CRF).

\subsection{Comparison to others}

We compare our best weak supervision strategy identified in the previous section. To generate full annotations, we regularise with FC-CRF the averaged predictions of the local (RFs) and the global (DeepLabV2 without multi-scaling) PAMs. We use these predicted full annotations to train different DCNNs: DeepLabv2-ResNet101 [4], PSPNet-ResNet50 and PSPNet-ResNet101 [39, 16] (see the supplementary material for details). We also test popular improvement strategies such as post-processing (CRF column in Tab. 2) and multi-scaling (MSC column in Tab. 2). In Tab. 2, we report the comparison results on the validation dataset of PASCAL VOC12. We achieve new state-of-theart performances in weakly supervised image segmentation with pixel-based annotations with a mIoU of 75.6 without and 75.7 with post-processing.

ScribbleSup [18] introduced a regularisation strategy for the predicted annotations based on superpixels and CRFs. In our framework, this corresponds to a regularised global PAM supervision strategy. However, their main improvement comes from iteratively retraining DeepLab, which is computationally expensive. Avoiding iterative retraining, our weak supervision strategy is faster and achieves lower gaps (3.0-3.7 vs. 5.6) and higher absolute values (75.7 vs. 63.1).

SimpleDoesIt [12] uses another pixel-based weak annotation modality: bounding boxes. However, their strategy is similar to ours in spirit. They designed a variant of GrabCut to predict partial annotations and segmentation proposal techniques to train DeepLab. We achieve a lower gap (3.7 vs. 5.1) and a higher mIoU (75.7 vs. 69.4).

Recently, NCL [32], CRF [33], KernelCut [33] established new state-of-the-art results by using traditional variational segmentation models within a deep learning framework to enable end-to-end training. These methods require implementing graph-cut algorithms within deep learning frameworks $[30,31]$ via new special layers that increase considerably the training time. Our strategy is more versatile because it does not require modifying the architecture of DCNNs. Our weak supervision strategy achieves higher absolute values (75.7 vs. 75.0) and reduces the gaps by a higher relative value (with MSC and without CRF: $64.2 \%$ (ours), $54.1 \%$ (NCL), $55.7 \%$ (CRF), $57.4 \%$ (KernelCut)).

\section{Conclusions and discussion}

Our study tackles the challenging problem of training semantic segmentation DCNNs in a weakly-supervised setting. We establish new experimental standards for this problem (see Tab. 1 and Fig. 2): measuring the gap by training solely on the weak annotations (1), quantifying the adversarial effect of annotation errors (2), and comparing different annotator models. This allows us to unravel a counter- 
Table 2. Comparison to state-of-the-art methods in weakly supervised semantic segmentation. All the values correspond to mIoU [\%] on the validation set of PASCAL VOC12. Weak + Baseline corresponds to training on the raw WAs. Full corresponds to training on the fully-annotated training set. Gap is the difference between the two. Weak + Strategy corresponds to training the Segmentation $D C N N$ using the weak supervision strategy shown in column Strategy. Remaining Gap computes the difference between full and weak supervision. Reduction (\%) is defined by (Full Gap - Remaining Gap)/Full Gap. $\left(^{*}\right)$ denotes methods using bounding box WAs. Best values are highlighted in bold.

\begin{tabular}{|c|c|c|c|c|c|c|c|c|c|}
\hline Strategy & Segmentation DCNN & MSC & CRF & Weak + Baseline & Full & Gap & Weak + Strategy & Remaining Gap & Reduction (\%) \\
\hline Ours & DeepLab-ResNet101 & - & - & 64.3 & 71.5 & 7.2 & 69.1 & 2.4 & $66.7 \%$ \\
\hline Ours & PSPNet-ResNet50 & - & 一 & 67.8 & 76.6 & 8.8 & 73.0 & 3.6 & $59.1 \%$ \\
\hline Ours & PSPNet-ResNet101 & - & - & 69.3 & 77.0 & 7.7 & 74.4 & 2.6 & $66.2 \%$ \\
\hline Ours & DeepLab-ResNet101 & - & $\checkmark$ & 66.4 & 73.4 & 7.0 & 70.5 & 2.9 & $58.6 \%$ \\
\hline Ours & PSPNet-ResNet50 & - & $\checkmark$ & 69.2 & 77.2 & 8.0 & 73.5 & 3.7 & $53.8 \%$ \\
\hline Ours & PSPNet-ResNet101 & - & $\checkmark$ & 70.7 & 77.5 & 6.8 & 74.7 & 2.8 & $58.8 \%$ \\
\hline NCL [32] & DeepLab-VGG16 & $\checkmark$ & - & 60.4 & 68.8 & 8.4 & 62.4 & 6.4 & $23.8 \%$ \\
\hline CRF [33] & DeepLab-VGG16 & $\checkmark$ & 一 & 60.4 & 68.8 & 8.4 & 64.4 & 4.4 & $47.6 \%$ \\
\hline KernelCut [33] & DeepLab-VGG16 & $\checkmark$ & - & 60.4 & 68.8 & 8.4 & 64.8 & 4.0 & $52.4 \%$ \\
\hline Ours & DeepLab-ResNet101 & $\checkmark$ & - & 65.6 & 73.7 & 8.1 & 70.8 & 2.9 & $64.2 \%$ \\
\hline NCL [32] & DeepLab-ResNet101 & $\checkmark$ & - & 69.5 & 75.6 & 6.1 & 72.8 & 2.8 & $54.1 \%$ \\
\hline CRF [33] & DeepLab-ResNet101 & $\checkmark$ & 一 & 69.5 & 75.6 & 6.1 & 72.9 & 2.7 & $55.7 \%$ \\
\hline KernelCut [33] & DeepLab-ResNet101 & $\checkmark$ & - & 69.5 & 75.6 & 6.1 & 73.0 & 2.6 & $57.4 \%$ \\
\hline Ours & PSPNet-ResNet50 & $\checkmark$ & - & 69.5 & 77.6 & 8.1 & 74.5 & 3.1 & $62.7 \%$ \\
\hline Ours & PSPNet-ResNet101 & $\checkmark$ & - & 71.3 & 79.2 & 7.9 & 75.6 & 3.6 & $54.4 \%$ \\
\hline ScribbleSup [18] & DeepLab-LargeFOV & $\checkmark$ & $\checkmark$ & - & 68.7 & - & 63.1 & 5.6 & - \\
\hline SimpleDoesIt* [12] & DeepLab-LargeFOV & $\checkmark$ & $\checkmark$ & - & 69.1 & - & 65.7 & 3.4 & - \\
\hline NCL [32] & DeepLab-VGG16 & $\checkmark$ & $\checkmark$ & 64.3 & 71.5 & 7.2 & 65.2 & 6.3 & $12.5 \%$ \\
\hline CRF [33] & DeepLab-VGG16 & $\checkmark$ & $\checkmark$ & 64.3 & 71.5 & 7.2 & 66.4 & 5.1 & $29.2 \%$ \\
\hline KernelCut [33] & DeepLab-VGG16 & $\checkmark$ & $\checkmark$ & 64.3 & 71.5 & 7.2 & 66.7 & 4.8 & $33.3 \%$ \\
\hline SimpleDoesIt* [12] & DeepLab-ResNet101 & $\checkmark$ & $\checkmark$ & - & 74.5 & - & 69.4 & 5.1 & - \\
\hline Ours & DeepLab-ResNet101 & $\checkmark$ & $\checkmark$ & 67.6 & 75.1 & 7.5 & 72.1 & 3.0 & $60.0 \%$ \\
\hline NCL [32] & DeepLab-ResNet101 & $\checkmark$ & $\checkmark$ & 72.8 & 76.8 & 4.0 & 74.5 & 2.3 & $42.5 \%$ \\
\hline CRF [33] & DeepLab-ResNet101 & $\checkmark$ & $\checkmark$ & 72.8 & 76.8 & 4.0 & 75.0 & 1.8 & $55.0 \%$ \\
\hline KernelCut [33] & DeepLab-ResNet101 & $\checkmark$ & $\checkmark$ & 72.8 & 76.8 & 4.0 & 75.0 & 1.8 & $55.0 \%$ \\
\hline Ours & PSPNet-ResNet50 & $\checkmark$ & $\checkmark$ & 69.9 & 77.8 & 7.9 & 74.6 & 3.2 & $59.5 \%$ \\
\hline Ours & PSPNet-ResNet101 & $\checkmark$ & $\checkmark$ & 71.8 & 79.4 & 7.6 & 75.7 & 3.7 & $51.3 \%$ \\
\hline
\end{tabular}

intuitive finding: averaging poor local predicted annotations with the baseline ones and reuse them for training a DCNN yields new state-of-the-art results $(\bullet, \square, \triangle)$. The fact that we achieve this without resorting to new cost-functions, regularization or architectures is a strength: it allows others to adapt our ideas to their setting and to extend the spectrum of strategies available for weakly-supervising DCNNs.

Motivations. This study started from two observations:

- DeepLabV2 trained solely on weak annotations (๑) already beats custom strategies like ScribbleSup.

- Additional annotations (62.5 times more for our data set) predicted by a model contain unavoidably errors. They have an adversarial effect on the resulting trained segmentation network $(\bullet, \square)$.

Insights. Our study allows tackling these questions:

- Have all the errors in the predicted annotations the same adversarial effect? No. After regularisation the local PFAs have a mIoU comparable to the baseline, but after training, the errors from the local PFAs have a higher negative impact on the accuracy on the validation set ( $\square$ ).

- What are generic strategies that can overcome the adversarial effect of predicted full annotations beyond the baseline, and how do they compare?

- Image-level regularisation using FC-CRF or Potts always helps $(\square, \square, \Delta, \square, \Delta)$, up to a certain extent: improving the accuracy after training by one point requires a $5 \times$ higher increase in the annotation quality (see linear trends in Fig. 2).

- Averaging local and global predicted annotations (๑) leads to better annotations than regularizing the predictions of the baseline $(\square, \mathbf{\Delta})$. The combined predictions are less adversarial for training a segmentation network and it achieves state-ofthe-art performances on this problem. 


\section{Appendix}

In this supplementary, we provide detailed architectures and training strategies, that we used to produce the results in the main paper.

\subsection{DeepLab: Architecture and Training}

Our strategy involves DeepLabV2 in two different tasks: (i) as a global PAM and (ii) as a final segmentation DCNN that is trained either on weak, predicted or human full annotations. For both tasks, we use the same architecture and training strategy. As a global PAM, we disable both multi-scaling and FC-CRF post-processing [13], unless mentioned otherwise. However, as the final segmentation DCNN, we assess the effect of both multi-scaling and post-processing by testing all combinations, see Tab. 2 in the main text.

The architecture of DeepLabV2 is based on ResNet101, connected with an Atrous Spatial Pyramid Pooling (ASPP) module with four branches (atrous rates $r \in$ $\{6,12,18,24\})$ and a bilinear up-sampling to match the input resolution. We initialise the model using the parameters of ResNet-101 pre-trained on ImageNet [26]. We use random initialisation for all other parameters. In particular, we avoid pre-training DeepLabV2 on any segmentation dataset such as MS-COCO [4]. We modify a publicly available TensorFlow implementation of DeepLabV2 [36]. Our training is as follows: $20 \mathrm{k}$ training iterations, a batch size of 10 , a momentum of 0.9 , a weight decay of 0.0005 and the following poly learning rate policy [4] $(1-\text { iter/maxiter })^{\text {power }}$, with a power of 0.9 and a learning rate of 0.000625 . We also enable data augmentation by randomly mirroring and scaling the input data. We calculate the loss after bilinear upsampling, (input resolution $321 \times 321$ ). We keep the same training policy for all annotation types (weak, predicted or human full annotations).

\subsection{PSPNet}

The architecture of the PSPNets [39] is based on either ResNet-50 or ResNet-101. We initialize the models using the parameters of the ResNets pre-trained on ImageNet [26]. Again, we avoid pre-training on any other data set, especially on segmentation data sets. We use a publicly available TensorFlow implementation of PSPNet [17].

Our training is as follows: $30 \mathrm{k}$ training iterations, a batch size of 16 , a $L^{2}-\mathrm{SP}$ [16] regularisation with parameters $\alpha=0.001$ and $\beta=0.0001$, a momentum optimizer with a momentum of 0.9 and the following poly learning rate policy $[4,39](1-\text { iter } / \text { maxiter })^{\text {power }}$, with a power of 0.9 and a learning rate of 0.01 . We use random rotation and random scaling for data augmentation with an image resolution of $480 \times 480$. We keep the same training policy for all annotation types (weak, predicted or human full annotations).

\subsection{Potts}

We select the regularisation parameter $\lambda$ by a grid search on a small subset of the training set. We use the parameters that achieve the highest mIoU on the predicted annotations: $\lambda=10$ when using the local or combined PAMs and $\lambda=$ 50 when only the global PAM is used. For the edge stop function, we use the parameter $\eta=0.01$.

\subsection{Fully Connected Conditional Random Field}

We selected the parameters of the FC-CRF by a grid search on the predicted full annotations, obtained from the combined model applied to a small subset of the training set. We use the same parameters for all experiments: $w_{1}=3$, $\sigma_{\alpha}=30, \sigma_{\beta}=5, w_{2}=5$ and $\sigma_{\gamma}=2$.

\section{References}

[1] A. Bearman, O. Russakovsky, V. Ferrari, and L. Fei-Fei. What's the Point: Semantic Segmentation with Point Supervision. In Computer Vision - ECCV 2016, pages 549-565, 2016. 1

[2] L. Beyer. PyDenseCRF. https://github.com/ lucasb-eyer/pydensecrf, 2018. 4

[3] L. Breiman. Random forests. Machine Learning, 45(1):532, Oct 2001. 3

[4] L.-C. Chen, G. Papandreou, I. Kokkinos, K. Murphy, and A. L. Yuille. DeepLab: Semantic Image Segmentation with Deep Convolutional Nets, Atrous Convolution, and Fully Connected CRFs. IEEE Transactions on Pattern Analysis and Machine Intelligence, 40(4):834-848, April 2018. 3, 7, 10

[5] J. Diebold, C. Nieuwenhuis, and D. Cremers. Midrange Geometric Interactions for Semantic Segmentation: Constraints for Continuous Multi-label Optimization. International Journal of Computer Vision, 117(3):199-225, 2016. 2

[6] M. Everingham, L. Van Gool, C. K. I. Williams, J. Winn, and A. Zisserman. The Pascal Visual Object Classes (VOC) Challenge. International Journal of Computer Vision, 88(2):303-338, Jun 2010. 1, 3

[7] D. A. Forsyth, J. Malik, M. M. Fleck, H. Greenspan, T. Leung, S. Belongie, C. Carson, and C. Bregler. Finding pictures of objects in large collections of images. In International workshop on object representation in computer vision, pages 335-360. Springer, 1996. 1

[8] A. Garcia-Garcia, S. Orts-Escolano, S. Oprea, V. VillenaMartinez, and J. Garcia-Rodriguez. A Review on Deep Learning Techniques Applied to Semantic Segmentation. arXiv preprint, pages 1-23, 2017. 1

[9] S. Hong, S. Kwak, and B. Han. Weakly Supervised Learning with Deep Convolutional Neural Networks for Semantic Segmentation: Understanding Semantic Layout of Images with Minimum Human Supervision. IEEE Signal Processing Magazine, 34:39-49, nov 2017. 2

[10] S. Hong, H. Noh, and B. Han. Decoupled Deep Neural Network for Semi-supervised Semantic Segmentation. In Pro- 
ceedings of the 28th International Conference on Neural Information Processing Systems - Volume 1, NIPS'15, pages 1495-1503, Cambridge, MA, USA, 2015. MIT Press. 1

[11] Z. Huang, X. Wang, J. Wang, W. Liu, and J. Wang. WeaklySupervised Semantic Segmentation Network With Deep Seeded Region Growing. In The IEEE Conference on Computer Vision and Pattern Recognition (CVPR), June 2018. 1

[12] A. Khoreva, R. Benenson, J. Hosang, M. Hein, and B. Schiele. Simple Does It: Weakly Supervised Instance and Semantic Segmentation. In The IEEE Conference on Computer Vision and Pattern Recognition (CVPR), July 2017. 1, 7,8

[13] P. Krähenbühl and V. Koltun. Efficient inference in fully connected CRFs with Gaussian edge potentials. In J. ShaweTaylor, R. S. Zemel, P. L. Bartlett, F. Pereira, and K. Q. Weinberger, editors, Advances in Neural Information Processing Systems 24, pages 109-117. Curran Associates, Inc., 2011. $2,3,4,10$

[14] A. Krizhevsky, I. Sutskever, and G. E. Hinton. ImageNet Classification with Deep Convolutional Neural Networks. In F. Pereira, C. J. C. Burges, L. Bottou, and K. Q. Weinberger, editors, Advances in Neural Information Processing Systems 25, pages 1097-1105. Curran Associates, Inc., 2012. 3

[15] L. Ladicky, C. Russell, P. Kohli, and P. H. S. Torr. Graph cut based inference with co-occurrence statistics. In K. Daniilidis, P. Maragos, and N. Paragios, editors, Computer Vision - ECCV 2010, pages 239-253, Berlin, Heidelberg, 2010. Springer Berlin Heidelberg. 2

[16] X. Li, Y. Grandvalet, and F. Davoine. Explicit inductive bias for transfer learning with convolutional networks. In International Conference on Machine Learning (ICML), pages 2830-2839, 2018. 7, 10

[17] X. Li, Y. Grandvalet, and F. Davoine. Training Reproduce of PSPNet. https: / / github.com/holyseven/ PSPNet-TF-Reproduce, 2019. 10

[18] D. Lin, J. Dai, J. Jia, K. He, and J. Sun. ScribbleSup: Scribble-Supervised Convolutional Networks for Semantic Segmentation. In The IEEE Conference on Computer Vision and Pattern Recognition (CVPR), June 2016. 1, 2, 3, 4, 5, 7, 8

[19] T.-Y. Lin, M. Maire, S. Belongie, J. Hays, P. Perona, D. Ramanan, P. Dollár, and C. L. Zitnick. Microsoft COCO: Common Objects in Context. In D. Fleet, T. Pajdla, B. Schiele, and T. Tuytelaars, editors, Computer Vision - ECCV 2014, pages 740-755. Springer International Publishing, 2014. 1

[20] C. Nieuwenhuis and D. Cremers. Spatially varying color distributions for interactive multilabel segmentation. IEEE Transactions on Pattern Analysis and Machine Intelligence, 35(5):1234-1247, 2013. 3

[21] C. Nieuwenhuis, E. Töppe, and D. Cremers. A survey and comparison of discrete and continuous multi-label optimization approaches for the Potts model. International Journal of Computer Vision, 104(3):233-240, 2013. 2, 3, 4

[22] G. Papandreou, L.-C. Chen, K. P. Murphy, and A. L. Yuille. Weakly- and semi-supervised learning of a deep convolutional network for semantic image segmentation. In The IEEE International Conference on Computer Vision (ICCV), December 2015. 1, 3
[23] D. Pathak, E. Shelhamer, J. Long, and T. Darrell. Fully Convolutional Multi-Class Multiple Instance Learning. ArXiv e-prints, Dec. 2014. 1

[24] G. Paul, J. Cardinale, and I. F. Sbalzarini. An alternating split Bregman algorithm for multi-region segmentation. In 2011 Conference Record of the Forty Fifth Asilomar Conference on Signals, Systems and Computers (ASILOMAR), pages 426-430, Nov 2011. 4

[25] C. Rother, V. Kolmogorov, and A. Blake. "GrabCut": Interactive Foreground Extraction Using Iterated Graph Cuts. ACM Trans. Graph., 23(3):309-314, Aug. 2004. 2, 3

[26] O. Russakovsky, J. Deng, H. Su, J. Krause, S. Satheesh, S. Ma, Z. Huang, A. Karpathy, A. Khosla, M. Bernstein, A. C. Berg, and L. Fei-Fei. ImageNet Large Scale Visual Recognition Challenge. International Journal of Computer Vision, 115(3):211-252, Dec 2015. 10

[27] J. Santner, T. Pock, and H. Bischof. Interactive multi-label segmentation. In R. Kimmel, R. Klette, and A. Sugimoto, editors, Computer Vision-ACCV 2010, pages 397-410, Berlin, Heidelberg, 2011. Springer Berlin Heidelberg. 3

[28] J. Santner, M. Unger, T. Pock, C. Leistner, A. Saffari, and $\mathrm{H}$. Bischof. Interactive texture segmentation using random forests and total variation. In Proceedings of the British Machine Vision Conference, pages 66.1-66.12. BMVA Press, 2009. doi:10.5244/C.23.66. 2, 3

[29] K. Simonyan and A. Zisserman. Very Deep Convolutional Networks for Large-Scale Image Recognition. In International Conference on Learning Representations (ICRL), pages 1-14, 2015. 3

[30] M. Tang. Pytorch version of regularized segmentation loss. https://github.com/meng-tang/rloss/ tree/master/pytorch, 2019. 7

[31] M. Tang. Regularized Losses (rloss) for Weaklysupervised CNN Segmentation. https://github. com/meng-tang/rloss, 2019. 7

[32] M. Tang, A. Djelouah, F. Perazzi, Y. Boykov, and C. Schroers. Normalized Cut Loss for Weakly-Supervised CNN Segmentation. In The IEEE Conference on Computer Vision and Pattern Recognition (CVPR), June 2018. 1, 3, 7, 8

[33] M. Tang, F. Perazzi, A. Djelouah, I. Ben Ayed, C. Schroers, and Y. Boykov. On regularized losses for weakly-supervised cnn segmentation. In The European Conference on Computer Vision (ECCV), September 2018. 3, 7, 8

[34] M. Unger, T. Pock, W. Trobin, D. Cremers, and H. Bischof. TVSeg - Interactive Total Variation Based Image Segmentation. In Proceedings of the British Machine Vision Conference, pages 40.1-40.10. BMVA Press, 2008. doi:10.5244/C.22.40. 2

[35] P. Vernaza and M. Chandraker. Learning Random-Walk Label Propagation for Weakly-Supervised Semantic Segmentation. In The IEEE Conference on Computer Vision and Pattern Recognition (CVPR), July 2017. 1

[36] Z. Wang. Deeplab-v2-ResNet-101-Tensorflow. https://github.com/zhengyang-wang/ Deeplab-v2--ResNet-101--Tensorflow, 2018. 4,10 
[37] Z. Wang and S. Ji. Smoothed dilated convolutions for improved dense prediction. In Proceedings of the 24th ACM SIGKDD International Conference on Knowledge Discovery \& Data Mining, pages 2486-2495. ACM, 2018. 4

[38] M. D. Zeiler and R. Fergus. Visualizing and understanding convolutional networks. In D. Fleet, T. Pajdla, B. Schiele, and T. Tuytelaars, editors, Computer Vision - ECCV 2014, pages 818-833, Cham, 2014. Springer International Publishing. 3

[39] H. Zhao, J. Shi, X. Qi, X. Wang, and J. Jia. Pyramid scene parsing network. In The IEEE Conference on Computer Vision and Pattern Recognition (CVPR), July 2017. 7, 10 